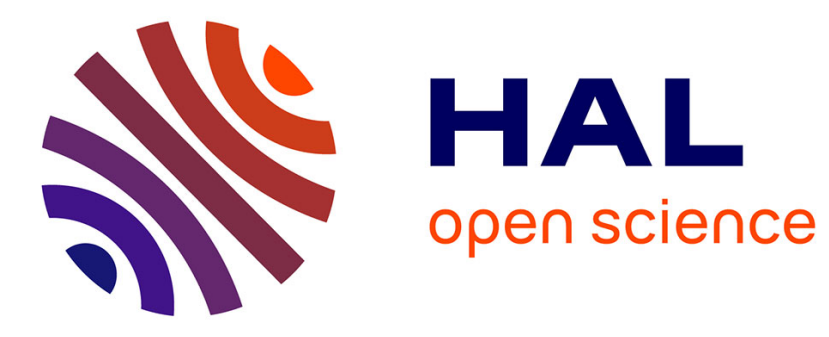

\title{
Training System for the Medical Procedure of Cannulation
}

Katherine Vera, Maria Chavarro, Andres Felipe Barco Santa, Élise Vareilles

\section{To cite this version:}

Katherine Vera, Maria Chavarro, Andres Felipe Barco Santa, Élise Vareilles. Training System for the Medical Procedure of Cannulation. IEEM 2018 - International Conference on Industrial Engineering and Engineering Management, Dec 2018, Bangkok, Thailand. pp.288 - 292, 10.1109/IEEM.2018.8607449 . hal-01969466

\section{HAL Id: hal-01969466 https://hal.science/hal-01969466}

Submitted on 7 Jan 2019

HAL is a multi-disciplinary open access archive for the deposit and dissemination of scientific research documents, whether they are published or not. The documents may come from teaching and research institutions in France or abroad, or from public or private research centers.
L'archive ouverte pluridisciplinaire HAL, est destinée au dépôt et à la diffusion de documents scientifiques de niveau recherche, publiés ou non, émanant des établissements d'enseignement et de recherche français ou étrangers, des laboratoires publics ou privés. 


\title{
Training System for the Medical Procedure of Cannulation
}

\author{
Katherine Vera ${ }^{1}$, Maria Chavarro ${ }^{1}$, Andrés F. Barco ${ }^{1}$, Élise Vareilles ${ }^{2}$ \\ ${ }^{1}$ Lidis Research Group, Universidad de San Buenaventura Cali, Cali, Colombia \\ ${ }^{2}$ Industrial Engineering Laboratory, Université de Toulouse, Mines Albi, Albi, France \\ Corresponding author: anfelbar@usbcali.edu.co
}

\begin{abstract}
This document presents a learning system architecture for the practice of the cannulation procedure for medical students in cardiovascular specialization. The architecture is composed of three modules that support the practice and learning of this surgical procedure; surgical instrumentation, hygiene and simulation of the cannulation. The architecture rests on different multimedia technologies that allow an autonomous practice but that may be supervised by the teacher. In the document are discussed the context, the need for tools for this medical practice, an architectural design of the proposed system and a comparison with the current practices in medicine programs.
\end{abstract}

Keywords - Training system, software architecture, cannulation technique, augmented reality, 3D models

\section{INTRODUCTION}

The present research is focused on the design and development of an interactive system that contributes in educational practices of medicine students in cardiovascular specialty by simulating the procedure of the technique of cannulation. This procedure is of vital importance as it is performed in most open-heart surgeries [1]. In few words, cannulation is the introduction of a tubular medical device in the human heart in order to allow the flow of blood during a surgery [2]. Being this one critical procedure for surgeries, and therefore fundamental in the learning of medical science, to the best of our knowledge there are no tools that allow an appropriate student practice of this procedure. This lack of tools for practice of students in cardiovascular specialty may generate problems of insecurities that are translated, potentially, in errors that affect the patients' well-being. Additionally, teachers also have problems in the teaching and supervision process given that it is learned, in essence, in real surgeries.

Although medicine has different tools of practice as are the mortal remains (corpses in general), digital devices, medicine applications and mannequins simulators of human body behavior, to the knowledge of the authors there is no tool focused on the practice of the technique of cannulation. In practicing tools, however, are reflected some of the characteristics necessary for a correct learning and practice of medical procedures, such as:
1. Feedback, which allows the student to account, how his actions have different reactions, positive and negative, in the practice he performing.

2. The follow-up or supervision of the teacher, who provides comments or corrections of the student's errors and helps to solve them.

3. Reuse, which allows the student to practice the times he deems convenient, so to find or correct his flaws during the practice.

4. The simulation of the procedure, which should be intuitive and attractive to the student in such a way that encourages him to repeat it.

Our interest in this research is to design and implement an appropriate architecture for an interactive system that involves multimedia technologies, such as augmented reality and reality virtual, for supervised learning of the medical procedure of cannulation. The characteristics of tools for practices that we have mentioned, serve as a guide for the design and development of our interactive system. In this document we present an architecture divided into three modules that seek to train the student in the different phases of the procedure; hygiene module, instrumentation module and simulation module of the cannulation procedure. We discuss the technologies involved in each module.

The article is divided into five sections. In Section II are presented related work of medicine practice tools that serves as references for the design of our system. In Section III the proposed system architecture is presented. In Section IV a comparison with the different tools for practice is presented. Concluding remarks are drawn in Section V.

\section{RELATED WORK}

The literature provides several tools focused on the learning of specific areas or procedures of medicine. In this section we present some of these tools in order to describe the elements of interaction implemented in them.

The first antecedent is a simulator in virtual reality that allows the training of a minimally invasive vascular surgery [2]. The 3D model used to simulate this surgery was built using files of a computerized tomography. This simulator has key elements of visualization interaction, such as 3D models, collisions handling, haptic device for feedback and a friendly interface. According to the authors' conclusions, the evaluation of this simulation tool is satisfactory in regard to two factors, computational 
performance and its interactive behavior. High quality 3D models and collision handling are constituent elements of our learning system.

As a second antecedent we present a virtual environment for the hand training and affinity of vision in a surgery of minimally invasive thoracic surgery [4]. This simulator, through a series of activities, trains the student on the positioning of hands at the moment of instrumental use. As illustrated in Fig. 1, the system analyzes the position of hands that, in principle, is unique for each type of instrument. It does so using a Leap Motion device for data capture. Although our research contemplates the use of such devices, the first approach tries to provide an affordable tool for the broad spectrum of university students and therefore their use is planned as future work.

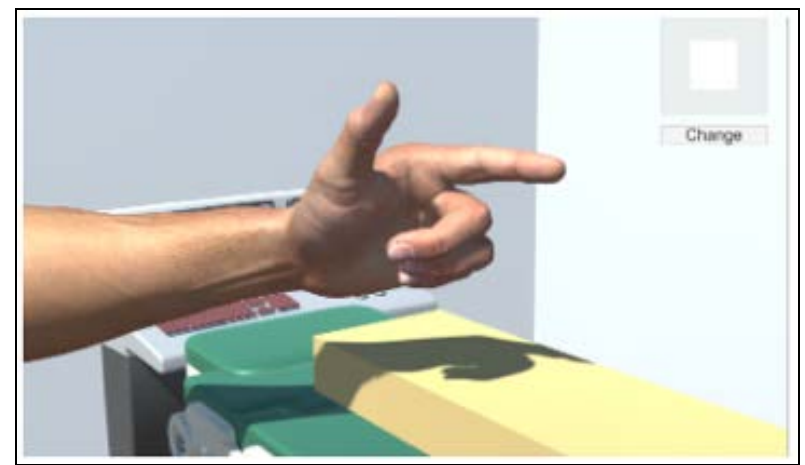

Fig. 1. Hand posture for the medical scalpel (taken from [4]).

In [5] is introduced a training simulator for the application of anesthesia. The researchers made use of haptic devices to simulate the force when applying the anesthesia and thus provide a realistic feedback. The simulator consists of three parts. The first part studies hygiene and the selection of anesthesia according to the patient's profile. The second part is responsible for simulating the application of anesthesia. Finally, in the third part, the user must monitor the patient during a simulated surgery. In Fig. 2 is presented a capture of the simulator.

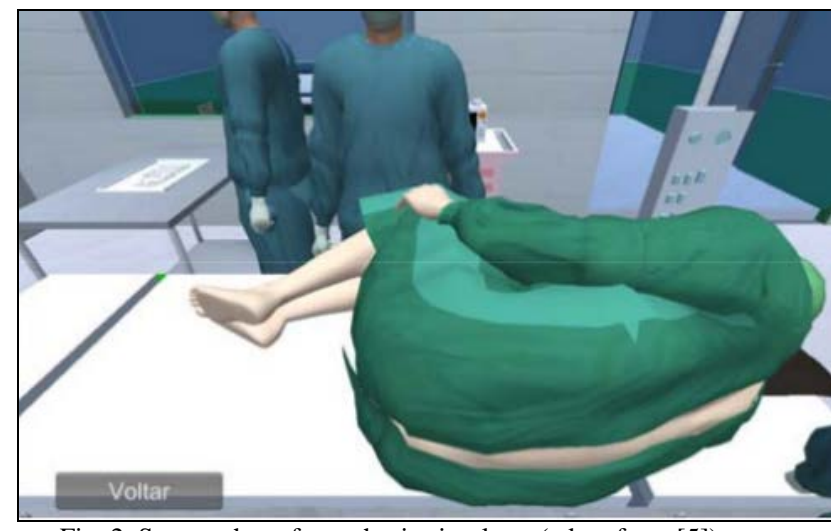

Fig. 2. Screen-shot of anesthesia simulator (taken from [5]).

As a last reference, we show a simulator for vascular surgery and an associated development kit to adapt its functionalities [6]. The authors focused on a tool that provides realism, optimization of computational resources and friendly interaction with the user. Scalability was also taken into account, to facilitate maintenance. Effects and animation, dynamics of soft body, multi-thread implementation and other functionalities make of it a robust and versatile tool. The interaction with this system, however, is mainly through the keyboard that impacts on the medical practice.

Most of the mentioned works seek to provide students who need it, new ways of learning and practice different procedures without resorting to expensive software or hardware. These tools then allow the students a constant training, reinforcing the skills of medicine students with the help of multimedia technology. In such works we identify common elements that guide our development; realism, intuitive interaction, reuse, supervision and modular division in order to guide the student by steps.

\section{SYSTEM'S INTERNAL DESIGN}

\section{A. General Scheme}

The investigative process was accompanied by Ethiana Dinas Muñoz, surgical instrumentalist specialized in cardiovascular surgeries. According to her comments, the difficulty of the technique of cannulation lies in the fact that its application has variants depending on characteristics associated with the patient (such as weight, size and age). Generally, students understand this in full only when practicing in surgeries with real patients. Additionally, according to the instrumentalist, the difficulty of the procedure is increased by hygiene practice and the specific instrumentation for the surgery. Consequently, the proposed architecture is divided into three modules implemented under different technologies.

The first module focuses on the hygiene that must be had before surgery and in the recognition of the patient, emphasizing the particular details of the patient that are important to the preparation of the surgery. The second module will consist of recognition and selection of appropriate instrumentation the cannulation technique. The last module simulates the surgical part, which consists of the procedure of the cannulation technique.

The architecture presents different interfaces for student use and teacher use. The student's interface allows the training (hygiene, instrumentation and procedure simulation) and the following of the progress. In the teacher interface, on the other hand, is visualized the interaction that performs, or was performed, the student with the system. This means that the practice is supervised by the teacher, although not necessarily in real-time. This facilitates to the teacher the following of the students' skills development and also to obtain statistics from groups of students. 


\section{B. Architectural View}

For the design of the system, and taking into account the elements used by the tools described in Section II, we have stablished they elements the system will exhibit:

1. 3D object modeling.

2. Feedbacks.

3. Teacher's supervision and interaction.

4. Record of execution.

5. Audio.

The last element, immersive audio, refers to reproduction of sounds commonly found in surgery rooms like instruments rubbing, heart beats and machine beats. The idea behind the audio is to promote the immersion when executing the cannulation simulation.

The architecture of the training system, implementing these aforementioned elements, is presented in Fig. 3. As we mentioned earlier, the student is capable of interact (train) and therefore visualize his training. On the other hand, the teacher is allowed only the simulation visualization, in real time or not. If the visualization is done in real time, the teacher has the option to provide comments both audio and text formats, in order to help or correct student's mistakes. Statistics of use of the tool, although not established yet, are possible thanks to persistent storage on a server Web.

The interface of each module involves different multimedia technologies. The hygiene module use text and sound and is evaluated with a questionnaire. The instrumentation module is implemented with augmented reality (see Fig. 4 and 5) and presents the specific instruments used for the cannulation procedure.

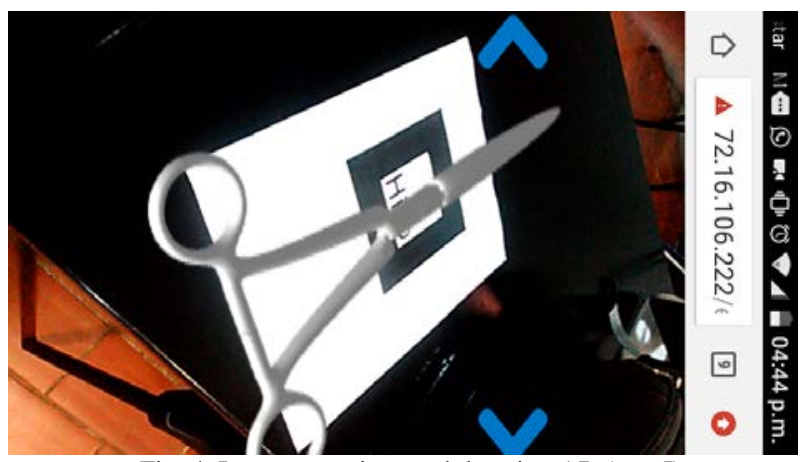

Fig. 4. Instrumentation module using AR (part I).

Finally, the module of the cannulation is implemented as a 3D environment with textures and collisions (realism).

\section{COMPARISON}

Around the world exist different academic programs at undergraduate and postgraduate levels related to medicine. These programs train students by making use of several technological tools each of them addressing a particular process or medical concept. Some of these tools

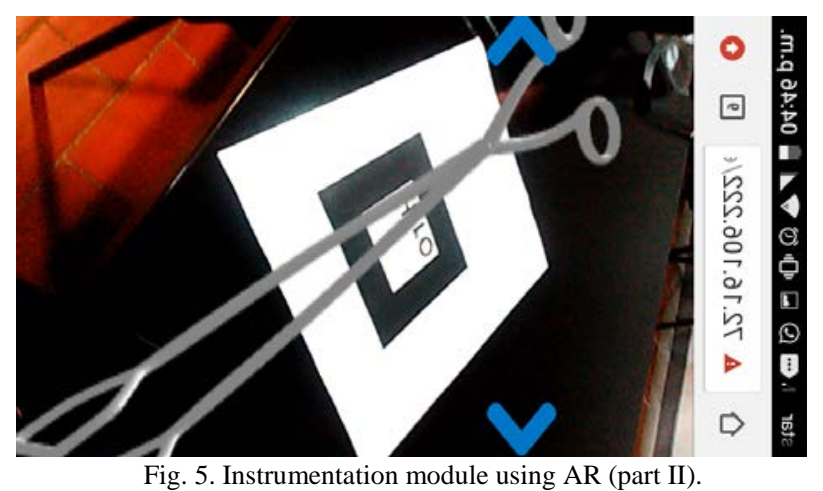

allow, and even force, teacher supervision, whereas other tools allow for autonomous practices. Although the objective of tools for practices is that of preparing students in the different medical procedures [7], none of them simulates the cannulation procedure. In what follows, we present the main tools used in medicine students training and expose the reasons why they, although useful for cannulation study, are not complete and thus used for the training of cannulation.

1. The first tool for medical learning are mobile applications. In these, there are a series of educational activities and simulators for different surgical procedures as well of concept of medicine in general (see Fig. 6 for one example from [8]). These applications are widely used and recommended by teachers, as they are accessible, reusable and require no teacher supervision. Optional supervision of students' training is not yet implemented in these application and, so far, the authors did not find applications for cannulation procedure practice.

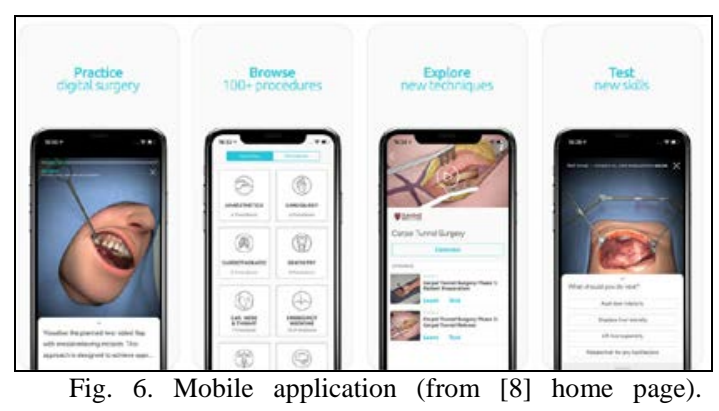

2. Advanced mannequins, on the other hand, present mechanisms that simulate the human body with materials, movements and reactions that mimic those of a living person [9] (see Fig. 6). These tools allow the simulation of a patient in different procedures like injectology and the taking of vital signs, among others. These devices, however, are very expensive and only the most advanced include flow of blood that allow a proper simulation of cannulation. 


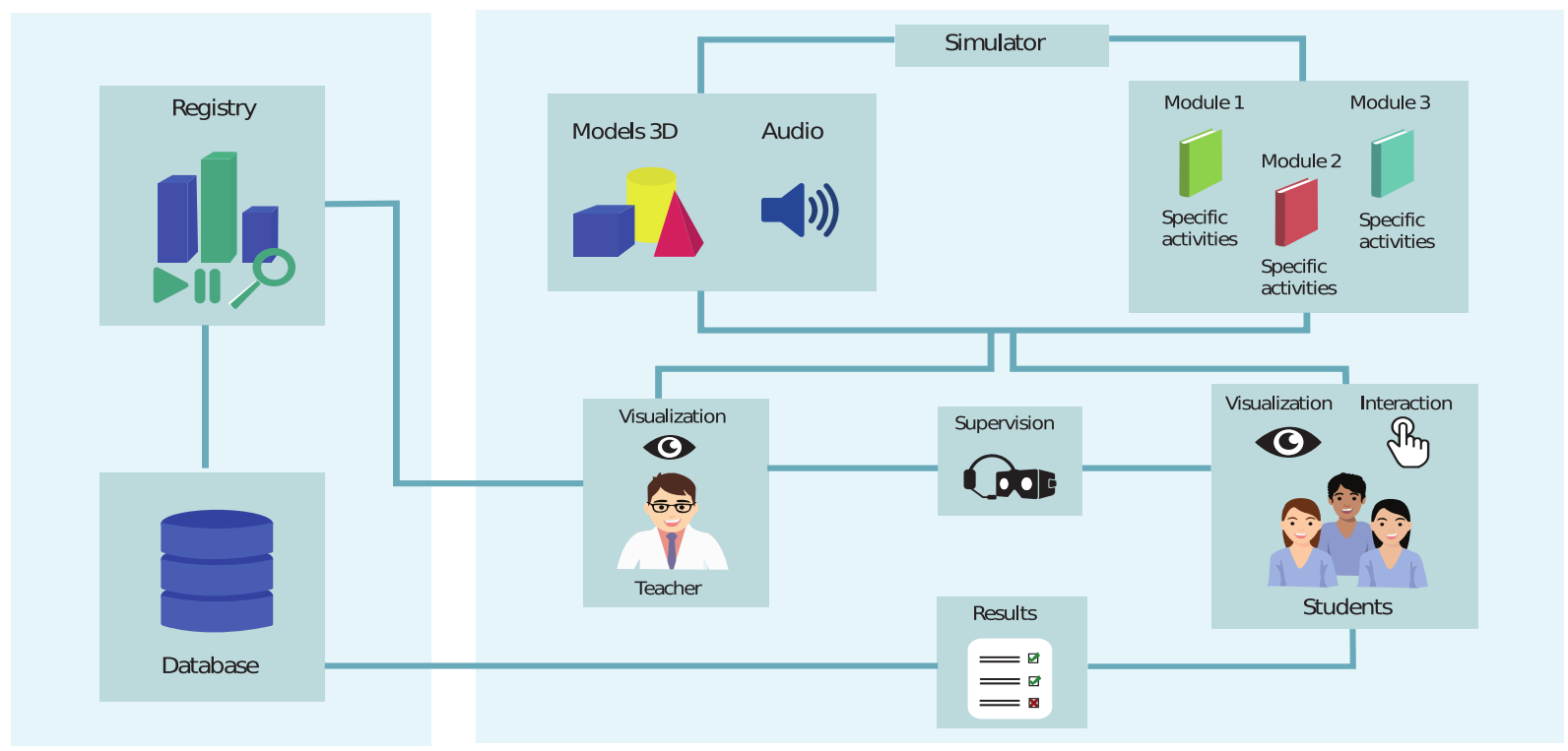

Fig. 7.Mannequin simulator (taken from [9]).

The device is reusable but supervised (given the risks of damage to the device).

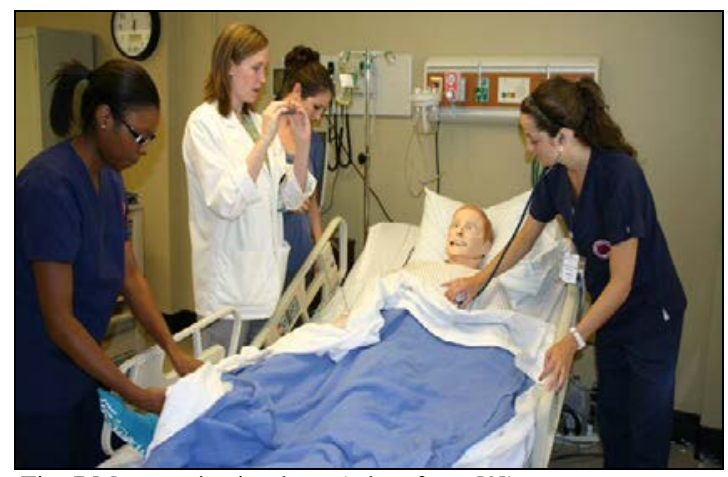

Fig. 7.Mannequin simulator (taken from [9]).

3. Electronic tables are an interesting alternative for studying the different systems that make up the human body, such as the one shown in Fig. 8, since they provide a very large amount of information and describe visually the different systems. They are used to teach anatomy to students, require a supervised practice and they are reusable [10]. The practice of cannulation is not possible as interaction is still limited to the touch screen.

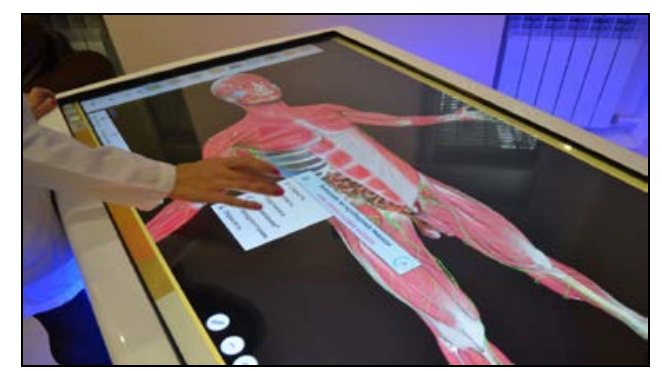

Fig. 8. Table for anatomy study (taken from [10]).
4. Finally, the human corpses used by most institutions to instruct in depth the anatomy of the body human. This practice must be carried out with the supervision of the teacher, in a limited interval of time and, although the corp is real, it does not provide de ideal feedback [11]. These 'tools' cannot be used to perform cannulation practice; blood does not circulate.

Evidently, no tool has all the elements needed for a complete medical practice, in particular for the cannulation procedure (exception of the living human body). However, the implementation of different elements for the medicine practice such as supervision of teaching, feedback, reuse and simulation of the cannulation technique may greatly help in certain learning and teaching aspects for both students and teachers. Ultimately, appropriated medical training translate in better patients' treatment in healthcare systems.

\section{CONCLUDING REMARKS}

This article presented a software architecture for the supervised training of the medical procedure of cannulation. Our architecture includes elements that are common to practicing tools used in medicine. The architecture is designed in such a way that the student can get feedback from the practice, even in real time, and is able to execute the simulation autonomously in the aim of reinforce knowledge. In the document we have presented the motivation behind our research, the relevant characteristics of existing training tools. We have discussed the proposed architecture and presented the reasons why other practice tools are not useful for the addressed medical procedure. This work then contributes to efforts to support training of the medical science. 


\section{ACKNOWLEDGMENT}

The authors want to thanks the surgery instrumentalist Etiana Dinas Muñoz, medical doctor at Clínica Amiga Cali-Colombia, for her guide through this work.

\section{REFERENCES}

[1] Dougherty, L., "Peripheral cannulation”, Nursing standard (Royal College of Nursing (Great Britain)), vol. 22, no. 52, pp. 49-56, 1987.

[2] Strauch, J.T., Spielvogel, D., Lauten, A., Lansman, S.L., McMurtry, K., Bodian, C.A., Griepp, R.B., “Axillary artery cannulation: Routine use in ascending aorta and aortic arch replacement”, Annals of Thoracic Surgery, vol. 78, no 1, pp. 103-108, 2004.

[3] S. Mi, Z. Hou, F. Yang, X. Xie and G. Bian, “A 3D virtual reality simulator for training of minimally invasive surgery”, in Annu. Engineering in Medicine and Biology Society (EMBC), Chicago, IL, US, Aug. 2014.

[4] J. Piedra, J. Ojeda, F. Quero and I. Piedra, "Virtual Environment for the Training of the Hands in Minimally Invasive Thoracic Surgery”, in Conf. Games and Virtual Worlds for Serious Applications (VS-Games), Barcelona, Spain, Sept. 2016.

[5] D. Gomes and L. Machado, "A Simulator for Regional Anesthesia Training”, in Symp. Virtual and Augmented Reality (SVR), Curitiba, Brazil, Nov. 2017.

[6] S. Mi, Z. Hou and F. Yang, "An 3D Interactive Virtual Reality Software Toolkit for Minimally Invasive Vascular Surgery” in Conf. Mechatronics and Automation (ICMA), Beijing, China, Aug. 2014.

[7] Tassos A. Mikropoulos, Antonis Natsis, "Educational virtual environments: A ten-year review of empirical research (1999-2009)”, Computers \& Education, vol. 56, no. 3, pp. 769-780. 2011.

[8] G.J. Bunogerane, K. Taylor, Y. Lin, A. Costas-Chavarri, "Using Touch Surgery to Improve Surgical Education in Low- and Middle-Income Settings: A Randomized Control Trial”, Journal of Surgical Education, vol. 75, no. 1, pp. 231-237, 2018.

[9] S. Changping, "Application of SimMan universal patient simulator in the teaching of medical nursing," in Proceedings 2011 International Conference on Human Health and Biomedical Engineering, Jilin, 2011, pp. 11721174.

[10] Ivaschenko, Anton and Gorbachenko, Nickolay and Kolsanov, Alexand, "Focused Visualization in Interactive Applications for Surgery Training”, in Conf. Creativity in Intelligent Technologies and Data Science, Volgograd, Russia, Sep. 2017.

[11] Ghosh Sanjib Kumar, "Cadaveric dissection as an educational tool for anatomical sciences in the 21st century". Anatomical Sciences Education, vol. 10(3), pp. 286-299. 2017. 